\title{
Joint Angle and Frequency Estimation in Linear Arrays Based on Covariance Reconstruction and ESPRIT
}

\author{
Shihong Chen $\mathbb{D}^{1},{ }^{1}$ Qingchang Tao, ${ }^{2}$ Zhongtian Yang, ${ }^{3}$ Xudong Wang, ${ }^{1}$ Sijia Liu, ${ }^{1}$ \\ and Wei $\mathrm{Xu}^{1}$ \\ ${ }^{1}$ Key Laboratory of Radar Imaging and Microwave Photonics, College of Electronic and Information Engineering, \\ Nanjing University of Aeronautics and Astronautics, Nanjing 211106, China \\ ${ }^{2}$ Department of Precision Instrument, State Key Laboratory of Precision Measurement Technology and Instruments, \\ Tsinghua University, Beijing 100084, China \\ ${ }^{3}$ College of Energy and Power Engineering, Nanjing University of Aeronautics and Astronautics, Nanjing 211106, China \\ Correspondence should be addressed to Shihong Chen; csh041230104@nuaa.edu.cn
}

Received 17 April 2021; Revised 13 June 2021; Accepted 29 June 2021; Published 9 July 2021

Academic Editor: Junpeng Shi

Copyright (C) 2021 Shihong Chen et al. This is an open access article distributed under the Creative Commons Attribution License, which permits unrestricted use, distribution, and reproduction in any medium, provided the original work is properly cited.

Joint angle and frequency estimation, one of the key technologies in wireless communication and radar science, has been extensively studied by scholars. For linear arrays, this paper proposes a joint angle and frequency estimation method based on covariance reconstruction and the estimation of signal parameters via rotational invariance techniques (CR-ESPRIT). We first use the received conjugate signal to reconstruct a covariance matrix. Then, we use the least squares-ESPRIT (LS-ESPRIT) algorithm to estimate the desired frequencies. Finally, we estimate the angles according to the reconstructed matrix. The proposed method can estimate signal parameters via automatic pairing and without an additional parameter pairing process under the condition of a uniform or a nonuniform array. Moreover, this method has high estimation accuracy, excellent and stable anti-noise performance, and strong algorithmic robustness. Through a computer simulation analysis, we can confirm the reliability and validity of the proposed parameter estimation method. A comparison with other methods further proves the performance advantages of the developed method. The method in this paper can be easily applied to many signal processing contexts, such as electronic reconnaissance and wireless communication.

\section{Introduction}

The joint angle and frequency estimation of received signals submerged in Gaussian white noise has important applications in wireless communication [1], audio and speech signal processing [2], and other fields [3, 4]. For example, in a wireless communication system, accurate and robust joint angle and frequency estimation can help provide better channel information, thereby improving the link quality and anti-interference ability of the system [1]. Especially in electronic reconnaissance [5-8], we often use the operating frequencies and directions of arrival (DOAs) [9-13] of noncooperative radar radiation source signals to describe the main parameters of radar signal characteristics [14-16]. Therefore, to effectively obtain the parameters of noncooperative radar source signals, it is necessary to study a joint DOA and frequency estimation method for such signals submerged in Gaussian white noise.

Regarding the joint DOA and frequency estimation of noisy signals, researchers worldwide have proposed various methods [16-22]. In 1986, Schmidt [17] proposed the multiple signal classification (MUSIC) algorithm for parameter estimation. Although the algorithm has good estimation performance, it has high computational complexity since it needs to search for spectral peaks to obtain the estimated values. Lemma et al. [18] presented a joint angle and frequency estimation method based on the multidimensional estimation of signal parameters via rotational invariance techniques (ESPRIT). Nevertheless, this algorithm has low parameter estimation accuracy under low 
signal-to-noise ratios (SNRs). To effectively improve the accuracy of estimated DOA and frequency results, in 2010, Wang proposed a joint angle and frequency estimation technique using multiple-delay outputs (MDJAFE) [16] based on the ESPRIT algorithm. However, this method cannot realize automatic parameter pairing when performing the joint estimation of signal parameters. Since the propagator method (PM) shows good performance in parameter estimation, it has attracted the attention of scholars. Sun et al. [19] proposed a joint DOA and frequency estimation based on the improved PM. Although the complexity of the algorithm is low and it can realize the automatic pairing of DOA and frequency parameter estimations, its parameter estimation accuracy is not high. Wang et al. [20] proposed an improved ESPRIT algorithm using the multidelay output of a uniform linear antenna (ULA). Although the algorithm's complexity is greatly reduced, this method is greatly affected by noise, and the estimation accuracy of this method is still very limited when the SNR is low. Based on the extended orthogonal matching pursuit (EOMP) algorithm, Gao et al. [21] proposed an approach to jointly estimate DOAs and frequency, whereas this method has high computational complexity. Xu et al. [22] proposed a joint DOA and narrowband source carrier frequency estimation method based on parallel factor (PARAFAC) analysis. The computational complexity of this method is relatively high, and the hardware cost is also high.

Due to the wide range of possible SNRs, frequency and DOA estimation algorithms have unstable anti-noise performance and limited estimation accuracy. We propose a method for the joint DOA and frequency estimation of signals submerged in Gaussian white noise. The algorithm involves a three-step estimation procedure. First, we preprocess the received signal. Second, we use the least squaresESPRIT (LS-ESPRIT) algorithm to estimate the frequency parameters of the signal. Finally, according to the unique relationship between the signal angle and its frequency, we estimate the DOAs. Computer simulations and comparisons with other methods prove the excellent performance of the proposed method.

The main contributions of our work can be summarized as follows:
(1) We improve upon the estimation process in [20]. Under the condition of a uniform or a nonuniform array, the method proposed in this paper can estimate the required parameters by performing automatic pairing without an additional parameter pairing process. Moreover, this method has good estimation accuracy, stable anti-noise performance, and robustness. Therefore, the method proposed in this paper is more suitable than other approaches for the parameter estimation of noncooperative radar radiation sources in an external field, which usually contains a complex electromagnetic environment.

(2) This paper proposes a joint angle and frequency estimation method based on covariance reconstruction and ESPRIT (CR-ESPRIT). Within the SNR range from $-15 \mathrm{~dB}$ to $15 \mathrm{~dB}$ (step: $2 \mathrm{~dB}$ ), its performance is better than that of the PM, the covariance reconstruction and propagator method (CR-PM), the ESPRIT method [16], and the improved ESPRIT method [20].

The remainder of this paper is structured as follows. The materials and methods are presented in Section 2; Section 3 contains the results and a discussion, and Section 4 is the summary of the paper.

Notations. $(\bullet)^{H},(\bullet)^{*},(\bullet)^{-1}$, and $(\bullet)^{+}$denote the conjugate transpose, complex conjugation, inverse, and Moore-Penrose inverse (pseudoinverse) operations, respectively. Matrices and vectors are represented by boldfaced capital letters and lowercase letters, respectively.

\section{Materials and Methods}

2.1. Signal Model. Consider an antenna array that consists of $M$ array elements arranged in a straight line at equal distances, where the distance between each pair of array elements is $d$ [23]. We suppose that there exist $K(K<M)$ farfield source narrowband signals (the center frequency is $f_{k}$ ), which are incident on the antenna array. Therefore, we can regard the signals as plane waves when they reach the array. Then, we can express the received signal of the $m$ th antenna as follows [24]:

$$
y_{m}(t)=\sum_{k=1}^{K} \exp \left(-j 2 \pi(m-1) \mathrm{d} f_{k}\left(\frac{\sin \left(\theta_{k}\right)}{c}\right)\right) s_{k}(t)+n_{m}(t), \quad m=1,2, \ldots, M
$$

where $s_{k}(t)$ is the $k$ th incident far-field source signal, $c$ is the speed of light $(\mathrm{m} / \mathrm{s}), \theta_{k}$ and $f_{k}$ are the DOA and frequency of the $k$ th signal, respectively, and $n_{m}(t)$ is the zero-mean additive white Gaussian noise on the $m$ th antenna. We can express the output signal of the linear array as

$$
\mathbf{Y}_{0}=\left[y_{1}(n) y_{2}(n), \ldots, y_{M}(n)\right]^{T}, \quad n=1,2, \ldots, N .
$$

We assume that the signal is uniformly sampled by a period that conforms to the Nyquist sampling rate and that the number of snapshots is $N$. Therefore, we can transform the signal model studied in this paper into a joint DOA and frequency estimation model for multiple source signals, where $N$ sampling points are obtained for each source signal.

We assume that the number of signal sources $K$ is known; thus, we can rewrite output state vector (2) in the following matrix form:

$$
\mathbf{Y}_{0}=\mathbf{A S}+\mathbf{N}_{0}
$$


where $\mathbf{S}=\left[\mathbf{s}_{1}, \mathbf{s}_{2}, \ldots, \mathbf{s}_{K}\right]^{T} \in C^{K \times N}, \mathbf{N}_{0}=\left[\mathbf{n}_{1}, \mathbf{n}_{2}, \ldots, \mathbf{n}_{M}\right]^{T}$ $\in C^{M \times N}$, and

$$
\mathbf{A}=\left[\begin{array}{cccc}
1 & 1 & \ldots & 1 \\
\exp \left(-j \alpha_{1}\right) & \exp \left(-j \alpha_{2}\right) & \ldots & \exp \left(-j \alpha_{K}\right) \\
\ldots & \ldots & \ldots & \ldots \\
\exp \left(-j(M-1) \alpha_{1}\right) & \exp \left(-j(M-1) \alpha_{2}\right) & \ldots & \exp \left(-j(M-1) \alpha_{K}\right)
\end{array}\right]
$$

In equation (4), $\alpha_{k}=2 \pi \mathrm{d} f_{k} \sin \left(\theta_{k}\right) / c, k=1, \ldots, K$. To realize the joint DOA and frequency estimation model, we take (P-1) delays $[25,26]$ for the signal received from the antenna arrays shown in Figure 1. In addition, we set $0<(P-1) \tau<1 / \max \left(f_{k}\right)$. Therefore, we can obtain the delay signal with the delay value $\tau$ as

$$
\begin{aligned}
y_{m}(t-\tau) & =\sum_{k=1}^{K} \exp \left(\frac{-j 2 \pi(m-1) \mathrm{d} f_{k} \sin \left(\theta_{k}\right)}{c}\right) s_{k}(t-\tau)+n_{m}^{\prime}(t) \\
& =\sum_{k=1}^{K} \exp \left(\frac{-j 2 \pi(m-1) \mathrm{d} f_{k} \sin \left(\theta_{k}\right)}{c}\right) s_{k}(t) \exp \left(-j 2 \pi f_{k} \tau\right)+n_{m}^{\prime}(t) .
\end{aligned}
$$

We can transform equation (5) into the following form:

$$
\mathbf{Y}_{1}=\mathbf{A} \Phi \mathbf{S}+\mathbf{N}_{1}
$$

where $\beta_{k}=2 \pi f_{k} \tau,(k=1,2, \ldots, K) \quad$ and $\quad \boldsymbol{\Phi}=\operatorname{diag}[\exp$ $\left.\left(-j \beta_{1}\right), \exp \left(-j \beta_{2}\right), \ldots, \exp \left(-j \beta_{K}\right)\right]$.

When the delay value is $p \tau$, we can express the delay signal as

$$
\begin{aligned}
y_{m}(t-p \tau) & =\sum_{k=1}^{K} \exp \left(\frac{-j 2 \pi(m-1) \mathrm{d} f_{k} \sin \left(\theta_{k}\right)}{c}\right) s_{k}(t-p \tau)+n_{m}^{\prime}(t) \\
& =\sum_{k=1}^{K} \exp \left(\frac{-j 2 \pi(m-1) \mathrm{d} f_{k} \sin \left(\theta_{k}\right)}{c}\right) s_{k}(t) \exp \left(-j 2 \pi f_{k} \tau p\right)+n_{m}^{\prime}(t) .
\end{aligned}
$$

Then, we can also express equation (7) as

$$
\mathbf{Y}_{p}=\mathbf{A} \boldsymbol{\Phi}^{p} \mathbf{S}+\mathbf{N}_{p}, \quad p=0,1,2, \ldots, P-1 .
$$

After reorganizing the equations, we can obtain the following expression:

$$
\mathbf{Y}=\left[\begin{array}{c}
\mathbf{Y}_{0} \\
\mathbf{Y}_{1} \\
\ldots \\
\mathbf{Y}_{P-1}
\end{array}\right]=\left[\begin{array}{c}
\mathbf{A} \\
\mathbf{A} \boldsymbol{\Phi} \\
\ldots \\
\mathbf{A} \boldsymbol{\Phi}^{P-1}
\end{array}\right] \mathbf{S}+\left[\begin{array}{c}
\mathbf{N}_{0} \\
\mathbf{N}_{1} \\
\ldots \\
\mathbf{N}_{P-1}
\end{array}\right]
$$

2.2. The Proposed Method. In this paper, inspired by the improved ESPRIT method [20], we propose a joint angle and frequency estimation method based on CR-ESPRIT. In a real space, the improved ESPRIT method is not suitable for complex electromagnetic environments. Due to the noncooperative characteristics of radiation sources, we generally believe that there is no prior information available regarding the parameters. Moreover, in a complex and harsh electromagnetic environment, the detected radiation source signals are very weak. Therefore, in a situation with a low SNR, the developed method not only needs to distinguish useful signals and noise effectively but also needs to have good estimation performance, noise immunity and robustness. Additionally, it also needs to have the ability to automatically pair the relevant parameters without an additional parameter pairing process under the condition of a uniform or a nonuniform array.

We first preprocess the received signal in Section 2.2.1. Second, we use the LS-ESPRIT algorithm to estimate the frequency parameters of the received signal in Section 2.2.2. Third, according to the relationship between the DOA and frequency in the signal model, we reconstruct the received signal, and then we estimate the DOAs in Section 2.2.3. In 


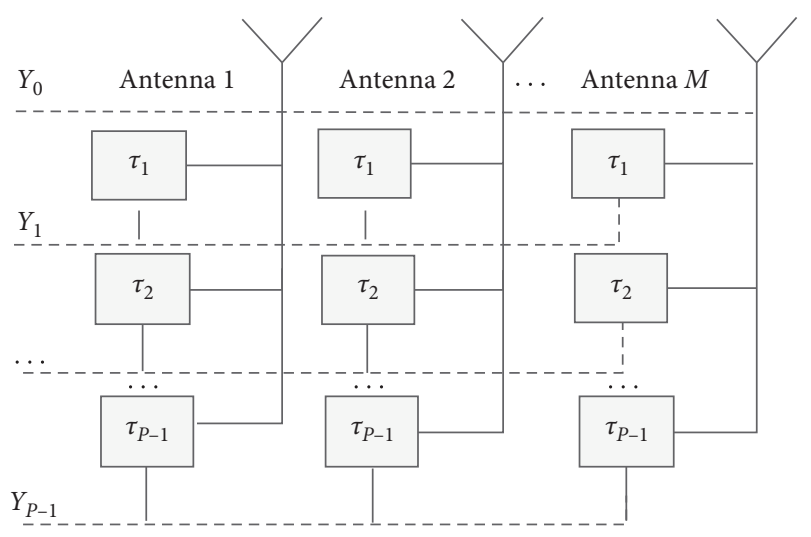

FIGURE 1: Received signals with multilevel delays.

Section 2.2.4, we provide the detailed steps of the proposed method. Finally, we provide the detailed steps of the proposed method under the condition of a nonuniform array in Section 2.2.5.

2.2.1. The Preprocessing Procedure. First, we obtain the covariance matrix $\mathbf{R}_{Y}=\mathbf{Y} \mathbf{Y}^{H}$ of the received signal. To make full use of the conjugate information contained in the received signal, we define the permutation matrix J [27]:

$$
\mathbf{J}=\left[\begin{array}{cccc}
0 & \ldots & 0 & 1 \\
\ldots & 0 & 1 & 0 \\
0 & \ldots & \ldots & \ldots \\
1 & 0 & \ldots & 0
\end{array}\right]_{P M \times P M}
$$

Therefore, we can construct the following matrix:

$$
\mathbf{R}_{J}=\mathbf{J}\left(\mathbf{Y}^{*}\right)\left(\mathbf{Y}^{*}\right)^{H} \mathbf{J}^{H}
$$

We add the covariance matrix $\mathbf{R}_{Y}$ and $\mathbf{R}_{J}$ from equation (11), and then we average them. The form of the obtained covariance matrix is shown as follows:

$$
\mathbf{R}=\frac{\mathbf{R}_{Y}+\mathbf{R}_{J}}{2}
$$

Through analysis, we can obtain that the new total covariance matrix $\mathbf{R}$ is a Hermitian matrix $(\mathrm{PM} \times \mathrm{PM})$ [28]. Therefore, we can apply eigenvalue decomposition, and then we can reconstruct the signal subspace $\mathbf{E}_{\mathrm{ss}}$. In a no-noise situation, $\mathbf{E}_{\mathrm{ss}}$ can be approximately expressed as

$$
\mathbf{E}_{\mathrm{ss}}=\left[\begin{array}{c}
\mathbf{A} \\
\mathbf{A} \Phi \\
\cdots \\
\mathbf{A} \Phi^{P-1}
\end{array}\right] \mathbf{F}
$$

where $\mathbf{F}$ is a full-rank matrix with $K \times K$ dimensions.

Remark 1. As mentioned earlier, the new total covariance matrix $\mathbf{R}$ is already a Hermitian matrix. According to Hermitian matrix characteristics, we assume that the diagonal matrix of the eigenvalues of $\mathbf{R}$ is $\mathbf{G}$. Then, there exists a unitary matrix $\mathbf{U}$, which assures $\mathbf{R U}=\mathbf{U G}$. Therefore, we can treat $\mathbf{R}$ as the unitary matrix $\mathbf{U}$ by using this correlation feature to further reduce the complexity of the proposed method and then propose a much lower complexity method.

2.2.2. Frequency Estimation. We define the following parameters:

$$
\begin{gathered}
\mathbf{E}_{1}=\left[\begin{array}{c}
\mathbf{A} \\
\mathbf{A} \Phi \\
\ldots \\
\mathbf{A} \Phi^{P-2}
\end{array}\right] \mathbf{F}, \\
\mathbf{E}_{2}=\left[\begin{array}{c}
\mathbf{A} \Phi \\
\mathbf{A} \Phi^{2} \\
\ldots \\
\mathbf{A} \Phi^{P-1}
\end{array}\right] \mathbf{F} .
\end{gathered}
$$

Therefore, equations (14) and (15) have the following relationship:

$$
\mathbf{E}_{2}=\left[\begin{array}{c}
\mathbf{A} \Phi \\
\mathbf{A} \Phi^{2} \\
\ldots \\
\mathbf{A} \Phi^{P-1}
\end{array}\right] \mathbf{F}=\left[\begin{array}{c}
\mathbf{A} \\
\mathbf{A} \Phi^{1} \\
\ldots \\
\mathbf{A} \Phi^{P-2}
\end{array}\right] \mathbf{F} \mathbf{F}^{-1} \boldsymbol{\Phi} \mathbf{F}=\mathbf{E}_{1} \mathbf{F}^{-1} \boldsymbol{\Phi} \mathbf{F}
$$

Let $\boldsymbol{\Psi}=\mathbf{F}^{-1} \boldsymbol{\Phi} \mathbf{F}$ and $\boldsymbol{\Psi}=\mathbf{E}_{1}^{+} \mathbf{E}_{2}$. According to the LSESPRIT algorithm, we can estimate $\Phi$ by the eigenvalue decomposition of $\boldsymbol{\Psi}$, and we can also estimate the matrix $\mathbf{F}^{-1}$ by the eigenvector of $\boldsymbol{\Phi}$. In a no-noise situation, we define

$$
\begin{aligned}
\widehat{\mathbf{F}}^{-1} & =\mathbf{F}^{-1} \boldsymbol{\Theta}, \\
\widehat{\boldsymbol{\Phi}} & =\boldsymbol{\Theta}^{-1} \boldsymbol{\Phi} \boldsymbol{\Theta},
\end{aligned}
$$

where $\boldsymbol{\Theta}$ is a fuzzy column matrix. Since $\boldsymbol{\Psi}$ and $\boldsymbol{\Phi}$ have the same eigenvalues, we can obtain the eigenvalues $\lambda_{k}(k=1,2, \ldots, K)$ from matrix $\Psi$. As shown in equation (6) $\left(\beta_{k}=2 \pi f_{k} \tau, k=1,2, \ldots, K\right)$, it is obvious that we can estimate the frequency parameter $\widehat{f}_{k}, k=1,2, \ldots, K$ :

$$
\widehat{f}_{k}=\frac{1}{2 \pi \tau} \operatorname{angle}\left(\lambda_{k}\right) \text {. }
$$


2.2.3. DOA Estimation. $\mathbf{A} \Phi^{P-1}$ has the following expression:

$$
\mathbf{A} \boldsymbol{\Phi}^{P-1}=\left[\begin{array}{cccc}
\exp \left(-j(P-1) \beta_{1}\right) & \exp \left(-j(P-1) \beta_{2}\right) & \ldots & \exp \left(-j(P-1) \beta_{K}\right) \\
\exp \left(-j \alpha_{1}\right) \exp \left(-j(P-1) \beta_{1}\right) & \exp \left(-j \alpha_{2}\right) \exp \left(-j(P-1) \beta_{2}\right) & \ldots & \exp \left(-j \alpha_{K}\right) \exp \left(-j(P-1) \beta_{K}\right) \\
\ldots & \ldots & \ldots & \ldots \\
\exp \left(-j(M-1) \alpha_{1}\right) \exp \left(-j(P-1) \beta_{1}\right) & \exp \left(-j(M-1) \alpha_{2}\right) \exp \left(-j(P-1) \beta_{2}\right) & \ldots & \exp \left(-j(M-1) \alpha_{K}\right) \exp \left(-j(P-1) \beta_{K}\right)
\end{array}\right] .
$$

According to the above estimation $\widehat{\mathbf{F}}^{-1}$, we can define the where following expression by reconstructing equation (13):

$$
\mathbf{E}_{Q}=\left[\begin{array}{c}
\mathbf{B} \\
\mathbf{B T} \\
\cdots \\
\mathbf{B T}^{M-1}
\end{array}\right] \Theta
$$

$$
\begin{aligned}
& \mathbf{B}=\left[\begin{array}{cccc}
1 & 1 & \ldots & 1 \\
\exp \left(-j \beta_{1}\right) & \exp \left(-j \beta_{2}\right) & \ldots & \exp \left(-j \beta_{K}\right) \\
\ldots & \ldots & \ldots & \ldots \\
\exp \left(-j(P-1) \beta_{1}\right) & \exp \left(-j(P-1) \beta_{2}\right) & \ldots & \exp \left(-j(P-1) \beta_{K}\right)
\end{array}\right] \\
& \mathrm{T}=\operatorname{diag}\left[\exp \left(-j \alpha_{1}\right), \exp \left(-j \alpha_{2}\right), \ldots, \exp \left(-j \alpha_{K}\right)\right] \in C^{K \times K}, \\
& \alpha_{k}=\frac{2 \pi \mathrm{d} f_{k} \sin \left(\theta_{k}\right)}{c}, \quad k=1,2, \ldots, K .
\end{aligned}
$$

According to reconstructed equation (20), we can use the method described below to estimate the DOA.

We define the following matrices:

$$
\begin{gathered}
\mathbf{E}_{Q 1}=\left[\begin{array}{c}
\mathbf{B} \\
\mathbf{B T} \\
\cdots \\
\mathbf{B T}^{M-2}
\end{array}\right] \boldsymbol{\Theta}, \\
\mathbf{E}_{Q 2}=\left[\begin{array}{c}
\mathbf{B T} \\
\mathbf{B T}^{2} \\
\cdots \\
\mathbf{B T}^{M-1}
\end{array}\right] \boldsymbol{\Theta} .
\end{gathered}
$$

We can also define a matrix $\mathbf{D}$ since $\mathbf{D}=\mathbf{E}_{\mathrm{Q} 1}^{+} \mathbf{E}_{\mathrm{Q} 2}$. Then, according to the definitions of $\mathbf{E}_{\mathrm{Q} 1}$ and $\mathbf{E}_{\mathrm{Q} 2}$, we can express $\mathbf{D}$ in a no-noise situation:

$$
\mathbf{D}=\Theta^{-1} \mathbf{T} \Theta .
$$

Therefore, we can take the diagonal elements of $\mathbf{D}$, and then, we can obtain $\omega_{k}(k=1,2, \ldots, K)$, where $\alpha_{k}=2 \pi \mathrm{d} f_{k} \sin \left(\theta_{k}\right) / c((k=1,2, \ldots, K)$, to obtain the estimation of the DOA:

$$
\widehat{\theta}_{k}=\arcsin \left(\frac{c}{2 \pi \widehat{f}_{k} d} \operatorname{angle}\left(\Phi_{k}\right)\right), \quad k=1,2, \ldots, K .
$$

2.2.4. The Steps of the Proposed Method. Thus far, we have given the complete process for automatically pairing DOA and frequency estimations in a linear array. The main steps required to implement the method proposed in this paper are as follows:

(i) Step 1: according to permutation matrix $\mathbf{J}$ and equation (12), we reconstruct the covariance matrix R.

(ii) Step 2: we apply eigenvalue decomposition to $\mathbf{R}$, and then we reconstruct the signal subspace $\mathbf{E}_{\mathrm{ss}}$. According to equations (14) and (15), we construct matrices $\mathbf{E}_{1}$ and $\mathbf{E}_{2}$, respectively.

(iii) Step 3: we use equation ( $\boldsymbol{\Psi}=\mathbf{E}_{1}^{+} \mathbf{E}_{2}$ ) for eigenvalue decomposition to obtain $\mathbf{F}^{-1}$ and $\widehat{\boldsymbol{\Phi}}$. Finally, we 
estimate the frequency parameter $\hat{f}_{k}$ according to equation (18).

(iv) Step 4: we can obtain matrix $\mathbf{E}_{\mathrm{Q}}$ according to the reconstruction of $\mathbf{E}_{\mathrm{ss}}$ in equation (13). Then, we can also construct matrices $\mathbf{E}_{Q 1}$ and $\mathbf{E}_{Q 2}$.

(v) Step 5: we calculate $\mathbf{D}=\mathbf{E}_{\mathrm{Q} 1}^{+} \mathbf{E}_{\mathrm{Q} 2}$ to obtain matrix $\mathbf{D}$. Finally, we estimate the DOA parameter $\widehat{\theta}_{k}$ according to equation (24).

2.2.5. The Condition of a Nonuniform Array. In this section, we first present the method proposed in this paper when the distances between the array elements are not equal. Then, we present the main steps for implementing the method in the case of a nonuniform array.
We assume that the first element $d_{1}=0$ and that the distance between the $m$ th element and the first element is $d_{\mathrm{m}}$. Then, we can transform equation (4) into the following form:

$$
\mathbf{A}_{1}=\left[\begin{array}{cccc}
1 & 1 & \ldots & 1 \\
\exp \left(-j d_{2} \eta_{1}\right) & \exp \left(-j d_{2} \eta_{2}\right) & \ldots & \exp \left(-j d_{2} \eta_{K}\right) \\
\ldots & \ldots & \ldots & \ldots \\
\exp \left(-j d_{M} \eta_{1}\right) & \exp \left(-j d_{M} \eta_{2}\right) & \ldots & \exp \left(-j d_{M} \eta_{K}\right)
\end{array}\right]
$$

where $\eta_{k}=2 \pi f_{k} \sin \left(\theta_{k}\right) / c(k=1,2, \ldots, K)$. At the same time, equation (19) undergoes the following transformation:

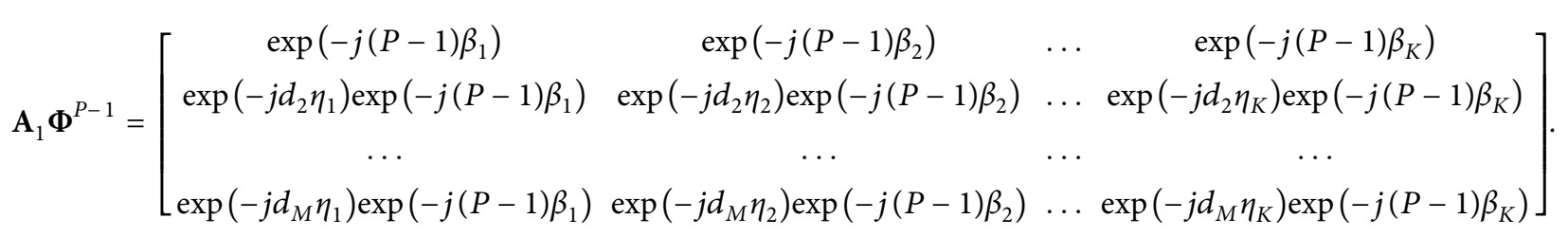

Similarly, we can reconstruct equation (13) and define the following expression:

$$
\mathbf{E}_{Q Q}=\left[\begin{array}{c}
\mathbf{B} \mathbf{J}_{1} \\
\mathbf{B J}_{2} \\
\cdots \\
\mathbf{B J}_{M}
\end{array}\right] \boldsymbol{\Theta},
$$

where $\quad \mathbf{J}_{m}=\operatorname{diag}\left[\exp \left(-j d_{m} \eta_{1}\right), \exp \left(-j d_{m} \eta_{2}\right)\right.$, $\left.\ldots, \exp \left(-j d_{m} \eta_{K}\right)\right] \in C^{K \times K}, m=1,2, \ldots, M$.

We define the following matrix:

$$
\begin{aligned}
& \mathbf{E}_{\mathrm{QQ1}}=\left[\begin{array}{c}
\mathbf{B J}_{1} \\
\mathbf{B J}_{2} \\
\cdots \\
\mathbf{B J}_{M-1}
\end{array}\right] \boldsymbol{\Theta}=\left[\begin{array}{c}
\mathbf{E}_{\mathrm{Q} 11} \\
\mathbf{E}_{\mathrm{QQ12}} \\
\cdots \\
\mathbf{E}_{\mathrm{QQ1}(M-1)}
\end{array}\right] \text {, } \\
& \mathbf{E}_{\mathrm{QQ2}}=\left[\begin{array}{c}
\mathbf{B J}_{2} \\
\mathbf{B J}_{3} \\
\ldots \\
\mathbf{B J}_{M}
\end{array}\right] \Theta=\left[\begin{array}{c}
\mathbf{E}_{\mathrm{QQ22}} \\
\mathbf{E}_{\mathrm{QQ23}} \\
\cdots \\
\mathbf{E}_{\mathrm{QQ2M}}
\end{array}\right] \text {. }
\end{aligned}
$$

We also define the matrix $\mathbf{Q}_{m}$, where $\mathbf{Q}_{m}=\left(\mathbf{E}_{\mathrm{QQ1}(m-1)}\right)^{+}\left(\mathbf{E}_{\mathrm{QQ2} 2 m}\right), m=2, \ldots, M$. Therefore, we can take the diagonal elements of $\boldsymbol{Q}_{m}$ and then obtain

$$
\boldsymbol{v}_{m}=\operatorname{diag}\left[\exp \left(-j\left(d_{m}-d_{m-1}\right) \eta_{1}\right), \exp \left(-j\left(d_{m}-d_{m-1}\right) \eta_{2}\right), \ldots, \exp \left(-j\left(d_{m}-d_{m-1}\right) \eta_{K}\right)\right] \in C^{K \times K}, \quad m=2, \ldots, M
$$

We sort the diagonal elements and then define the following matrix:

$$
\mathbf{V}=\left[\begin{array}{cccc}
1 & 1 & \ldots & 1 \\
\exp \left(-j\left(d_{2}-d_{1}\right) \eta_{1}\right) & \exp \left(-j\left(d_{2}-d_{1}\right) \eta_{2}\right) & \ldots & \exp \left(-j\left(d_{2}-d_{1}\right) \eta_{K}\right) \\
\ldots & \ldots & \ldots & \ldots \\
\exp \left(-j\left(d_{m}-d_{m-1}\right) \eta_{1}\right) & \exp \left(-j\left(d_{m}-d_{m-1}\right) \eta_{2}\right) & \ldots & \exp \left(-j\left(d_{m}-d_{m-1}\right) \eta_{K}\right)
\end{array}\right]
$$

According to equation (30), we can obtain the estimation of the DOA: 


$$
\widehat{\theta}_{k}=\frac{1}{M-1} \sum_{m=2}^{M} \arcsin \left(\frac{c}{2 \pi \widehat{f}_{k}\left(d_{m}-d_{m-1}\right)} \operatorname{angle}\left(\mathbf{V}_{m k}\right)\right), \quad(k=1,2, \ldots, K) .
$$

The main steps for implementing the method in this paper under the condition of a nonuniform array are as follows:

(i) Step 1: according to equations (10), (12), and (25), we reconstruct the new covariance matrix.

(ii) Step 2: we apply eigenvalue decomposition to the new covariance matrix, and then we reconstruct the signal subspace $\mathbf{E}_{\mathrm{ss}}$. According to equations (14) and (15), we construct matrices $\mathbf{E}_{1}$ and $\mathbf{E}_{2}$, respectively.

(iii) Step 3: we use equation $\left(\boldsymbol{\Psi}=\mathbf{E}_{1}^{+} \mathbf{E}_{2}\right)$ to perform eigenvalue decomposition and obtain $\mathbf{F}^{-1}$ and $\widehat{\boldsymbol{\Phi}}$. Finally, we estimate the frequency parameter $\widehat{f}_{k}$ according to equation (18).

(iv) Step 4: we can obtain the matrix $\mathbf{E}_{Q Q}$ according to equation (27). Then, we can also construct matrices $\mathbf{E}_{Q Q 1}$ and $\mathbf{E}_{Q Q 2}$.

(v) Step 5: we can obtain matrix $\mathbf{V}$ according to equation (30). Finally, we estimate the DOA parameter $\widehat{\theta}_{k}$ according to equation (31).

\section{Results and Discussion}

\subsection{Performance Analysis of the Proposed Method}

3.1.1. Method Complexity. In this section, we focus on the performance analysis with respect to complexity.
Complexity is mainly measured by the number of complex multiplications and the running time required by a given method. For the ESPRIT method in [16], the complexity is $\mathrm{O}\left(M^{2} P^{2} N+M^{3} P^{3}+2 K^{2} M(P-1)+8 K^{3}+2 K^{2}(M-1)\right)$.

For the improved ESPRIT method in [20], the complexity required to calculate the covariance matrix $\mathbf{R}_{\mathrm{Y}}$ is $\mathrm{O}\left(M^{2} P^{2} N\right)$. The complexity required for eigenvalue decomposition is $\mathrm{O}\left(M^{3} P^{3}\right)$. The complexity of calculating $\boldsymbol{\Psi}=$ $\mathbf{E}_{1}^{+} \mathbf{E}_{2}$ is $\mathrm{O}\left(2 K^{2} M(P-1)+2 K^{3}\right)$. Then, the eigenvalue decomposition complexity of $\boldsymbol{\Psi}=\mathbf{E}_{1}^{+} \mathbf{E}_{2}$ is $\mathrm{O}\left(K^{3}\right)$. When estimating the DOA, the complexity is $\mathrm{O}\left(2 K^{3}+2 K^{2}\right.$ $(M-1) P)$. Therefore, the complexity of the improved ESPRIT method is $\mathrm{O}\left(M^{2} P^{2} N+M^{3} P^{3}+2 K^{2} M(P-1)+\right.$ $\left.5 K^{3}+2 K^{2}(M-1) P\right)$.

For the proposed method, the preprocessing complexity is $\mathrm{O}\left(M^{2} P^{2} N+M^{3} P^{3}\right)$. The complexity of frequency estimation is $\mathrm{O}\left(2 K^{2} M(P-1)+3 K^{3}\right)$. In addition, the complexity of DOA estimation is $\mathrm{O}\left(2 K^{3}+2 K^{2}(M-1) P\right)$. Therefore, the complexity of the proposed method is $\mathrm{O}\left(M^{2} P^{2} N+M^{3} P^{3}+2 K^{2} M(P-1)\right.$ $\left.+5 K^{3}+2 K^{2}(M-1) P+1\right)$. It should be noted that in the preprocessing of this paper, we only need to calculate the covariance matrix $\mathbf{R}_{\mathrm{Y}}$ and the eigenvalue decomposition, which means that we do not require additional calculations to construct the matrix $\mathbf{R}_{\mathrm{J}}$.

The reason is that, according to equation (11), $\mathbf{R}_{\mathrm{J}}$ has the following expression:

$$
\begin{aligned}
& \mathbf{R}_{\mathbf{J}}=\mathbf{J Y}^{*} \mathbf{Y}^{* H} \mathbf{J}^{H}=\left[\begin{array}{cccc}
0 & \ldots & 0 & 1 \\
\ldots & 0 & 1 & 0 \\
0 & \ldots & \ldots & \ldots \\
1 & 0 & \ldots & 0
\end{array}\right]\left[\begin{array}{c}
\mathbf{Y}_{0} \\
\mathbf{Y}_{1} \\
\ldots \\
\mathbf{Y}_{P-1}
\end{array}\right]^{*}\left[\begin{array}{c}
\mathbf{Y}_{0} \\
\mathbf{Y}_{1} \\
\ldots \\
\mathbf{Y}_{P-1}
\end{array}\right]^{* H}\left[\begin{array}{cccc}
0 & \ldots & 0 & 1 \\
\ldots & 0 & 1 & 0 \\
0 & \ldots & \ldots & \ldots \\
1 & 0 & \ldots & 0
\end{array}\right]=\left[\begin{array}{c}
\mathbf{Y}_{P-1}^{*} \\
\ldots \\
\mathbf{Y}_{1}^{*} \\
\mathbf{Y}_{0}^{*}
\end{array}\right]\left[\begin{array}{llll}
\mathbf{Y}_{0} & \mathbf{Y}_{1} & \ldots & \mathbf{Y}_{P-1}
\end{array}\right]\left[\begin{array}{cccc}
0 & \ldots & 0 & 1 \\
\ldots & 0 & 1 & 0 \\
0 & \ldots & \ldots & \ldots \\
1 & 0 & \ldots & 0
\end{array}\right], \\
& =\left[\begin{array}{cccc}
\mathbf{Y}_{P-1}^{*} \mathbf{Y}_{0} & \mathbf{Y}_{P-1}^{*} \mathbf{Y}_{1} & \ldots & \mathbf{Y}_{P-1}^{*} \mathbf{Y}_{P-1} \\
\ldots & \ldots & \ldots & \ldots \\
\mathbf{Y}_{1}^{*} \mathbf{Y}_{0} & \mathbf{Y}_{1}^{*} \mathbf{Y}_{1} & \ldots & \mathbf{Y}_{1}^{*} \mathbf{Y}_{P-1} \\
\mathbf{Y}_{0}^{*} \mathbf{Y}_{0} & \mathbf{Y}_{0}^{*} \mathbf{Y}_{1} & \ldots & \mathbf{Y}_{0}^{*} \mathbf{Y}_{P-1}
\end{array}\right]\left[\begin{array}{cccc}
0 & \ldots & 0 & 1 \\
\ldots & 0 & 1 & 0 \\
0 & \ldots & \ldots & \ldots \\
1 & 0 & \ldots & 0
\end{array}\right]=\left[\begin{array}{cccc}
\mathbf{Y}_{P-1}^{*} \mathbf{Y}_{P-1} & \ldots & \mathbf{Y}_{P-1}^{*} \mathbf{Y}_{1} & \mathbf{Y}_{P-1}^{*} \mathbf{Y}_{0} \\
\ldots & \ldots & \ldots & \ldots \\
\mathbf{Y}_{1}^{*} \mathbf{Y}_{P-1} & \ldots & \mathbf{Y}_{1}^{*} \mathbf{Y}_{1} & \mathbf{Y}_{1}^{*} \mathbf{Y}_{0} \\
\mathbf{Y}_{0}^{*} \mathbf{Y}_{P-1} & \ldots & \mathbf{Y}_{0}^{*} \mathbf{Y}_{1} & \mathbf{Y}_{0}^{*} \mathbf{Y}_{0}
\end{array}\right],
\end{aligned}
$$

while the covariance matrix $\mathbf{R}_{\mathrm{Y}}$

$$
\mathbf{R}_{\mathbf{Y}}=\mathbf{Y Y}^{H}=\left[\begin{array}{c}
\mathbf{Y}_{0} \\
\mathbf{Y}_{1} \\
\ldots \\
\mathbf{Y}_{P-1}
\end{array}\right]\left[\begin{array}{c}
\mathbf{Y}_{0} \\
\mathbf{Y}_{1} \\
\ldots \\
\mathbf{Y}_{P-1}
\end{array}\right]^{H}=\left[\begin{array}{c}
\mathbf{Y}_{0} \\
\mathbf{Y}_{1} \\
\ldots \\
\mathbf{Y}_{P-1}
\end{array}\right]\left[\begin{array}{llll}
\mathbf{Y}_{0}^{*} & \mathbf{Y}_{1}^{*} & \ldots & \mathbf{Y}_{P-1}^{*}
\end{array}\right]=\left[\begin{array}{cccc}
\mathbf{Y}_{0} \mathbf{Y}_{0}^{*} & \mathbf{Y}_{0} \mathbf{Y}_{1}^{*} & \ldots & \mathbf{Y}_{0} \mathbf{Y}_{P-1}^{*} \\
\mathbf{Y}_{1} \mathbf{Y}_{0}^{*} & \mathbf{Y}_{1} \mathbf{Y}_{1}^{*} & \ldots & \mathbf{Y}_{1} \mathbf{Y}_{P-1}^{*} \\
& \ldots & \ldots & \ldots \\
\mathbf{Y}_{P-1} \mathbf{Y}_{0}^{*} & \mathbf{Y}_{P-1} \mathbf{Y}_{1}^{*} & \ldots & \mathbf{Y}_{P-1} \mathbf{Y}_{P-1}^{*}
\end{array}\right]
$$


By observing equations (32) and (33), we find that through simple moment transformation, we can transform the matrix $\mathbf{R}_{\mathrm{Y}}$ into the matrix $\mathbf{R}_{\mathrm{J}}$. Therefore, when preprocessing, we do not need additional complex multiplications to reconstruct the matrix $\mathbf{R}_{\mathrm{J}}$.

For the PM, the complexity of frequency estimation is $\mathrm{O}\left(M^{2} P^{2} N+4 K^{3}+M^{2} P^{2} K+P M K^{2}+2 K^{2}(M-K)\right)$. In addition, the complexity of DOA estimation is $\mathrm{O}\left(K^{2}(M-\right.$ $\left.K)+2 K^{3}+2 K^{2}(M-1)\right)$. Therefore, the complexity of the $\mathrm{PM}$ is $\mathrm{O}\left(M^{2} P^{2} N+6 K^{3}+M^{2} P^{2} K+P M K^{2}+3 K^{2}(M-K)\right.$ $\left.+2 K^{2}(M-1)\right)$. The CR method is also applicable to the PM. Therefore, the complexity of the CR-PM is $\mathrm{O}\left(M^{2} P^{2} N+\right.$ $\left.6 K^{3}+M^{2} P^{2} K+P M K^{2}+3 K^{2}(M-K)+2 K^{2}(M-1)\right)$.

Figures 2 and 3 present the complexity comparison of these algorithms versus the number of signal sources $K$ and the number of snapshots $N$ with $M=12$ and $P=3$, respectively. Table 1 compares the running time of these algorithms under the condition of an i7-8550U CPU with $K=3, N=200$, and 2000 Monte Carlo simulations. Figures 2 and 3 show that the complexity of the method proposed in this paper is almost the same as that of the ESPRIT method in [16] and that of the improved ESPRIT method in [20] and is much higher than that of the PM and that of the CR-PM. In addition, the running time of the proposed method does not increase much. Moreover, through subsequent analysis, within the SNR range from $-15 \mathrm{~dB}$ to $-1 \mathrm{~dB}$, the advantages of the proposed method are more obvious. In particular, when $\mathrm{SNR}=-15 \mathrm{~dB}$, compared with the improved ESPRIT method, the frequency estimation accuracy of the method proposed in this paper is an approximately $25.50 \%$ improvement; the DOA estimation accuracy of the method proposed in this paper is an approximately $31.95 \%$ improvement. Therefore, we can confirm that by increasing the utilization of the originally received data, we can improve the parameter estimation accuracy and the noise robustness of the proposed method.

3.1.2. The Advantages of the Proposed Method. In this section, we summarize the advantages of the proposed method in this paper as follows:

(1) Under the condition of a uniform or a nonuniform array, the method can effectively estimate the DOAs and frequencies of source signals. It can also realize automatic pairing without an additional parameter pairing process since the method has the same fuzzy column matrix for both parameters.

(2) For incoherent signal sources whose angles are close together, this method can perform effective identification and parameter estimation.

(3) Compared with those of the PM, the CR-PM, the ESPRIT method [16], and the improved ESPRIT method [20], the frequency and DOA estimation accuracies of the proposed method are greatly improved, and this method has superior estimation performance. Moreover, the proposed method has better anti-noise performance and stronger robustness.
3.2. Numerical Simulation. In the simulation, we assume that the array receives signals emitted by $K$ incoherent farfield sources. We also use the root mean square error (RMSE) metric to evaluate the DOA and frequency estimation performances of the proposed method; we define the RMSEs as

$$
\begin{aligned}
\mathrm{RMSE}_{\mathrm{DOA}} & =\frac{1}{K} \sum_{k=1}^{K} \sqrt{\frac{1}{L} \sum_{l}^{L}\left(\widehat{\theta}_{k, l}-\theta_{k}\right)^{2}}, \\
\mathrm{RMSE}_{\text {frequency }} & =\frac{1}{K} \sum_{k=1}^{K} \sqrt{\frac{1}{L} \sum_{l}^{L}\left(\hat{f}_{k, l}-f_{k}\right)^{2}},
\end{aligned}
$$

where $\hat{\theta}_{k, l}$ and $\hat{f}_{k, l}$ are the estimated values of $\theta_{k}$ and $f_{k}$, respectively, in the $l$ th Monte Carlo simulation and $L$ is the number of Monte Carlo simulations. In this paper, we set $L=2000$.

3.2.1. Performance Analysis of the Proposed Method in a Uniform Array. In this section, we assume that the array receives signals emitted by three incoherent far-field sources. The DOAs and operating frequencies of the signals are $\left(\theta_{1}\right.$, $\left.f_{1}\right)=\left(15^{\circ}, 1 \mathrm{MHz}\right),\left(\theta_{2}, f_{2}\right)=\left(40^{\circ}, 2.1 \mathrm{MHz}\right)$, and $\left(\theta_{3}, f_{3}\right)=\left(50^{\circ}\right.$, $3.1 \mathrm{MHz})$. SNR $=0 \mathrm{~dB}, M=12$ is the number of array elements, $P=3$ is the number of delay values, $d=50$ denotes the distances between the array elements, and $N=400$ and $K=3$ are the numbers of snapshots and signal sources, respectively. The scatter diagram of the joint frequency and DOA estimation of the proposed method in this paper is shown in Figure 4 . Figure 4 shows that the proposed method is efficient in estimating the frequency and DOA results for a uniform array.

3.2.2. Performance Analysis under Different Numbers of Array Elements $M$. We set $d=50 \mathrm{~m}, K=3, P=3$, and $N=400$. We also set different numbers of array elements $(M=8,12$, and 16). The SNR range is from $-15 \mathrm{~dB}$ to $15 \mathrm{~dB}$ (step: $2 \mathrm{~dB}$ ), and the RMSEs of the frequency and DOA estimates of the method proposed in this paper are shown in Figures 5 and 6 , respectively.

We can see from Figures 5 and 6 that the method proposed in this paper can achieve high estimation performance within the SNR range of $-15 \mathrm{~dB}$ to $15 \mathrm{~dB}$ (step: $2 \mathrm{~dB}$ ) under different numbers of array elements. The estimation performance is stable under the condition of a low SNR. Moreover, we can see that the SNR has a great impact on the estimation accuracies of the frequency and DOA. The higher the SNR is, the higher the parameter estimation accuracies of the method for these two parameters. With the increase in the number of array elements, the DOA and frequency estimation accuracies of the method proposed in this paper improve. Furthermore, the RMSEs of the proposed method are greatly reduced. This is because as the number of array elements increases, the space diversity gain increases [29]. 


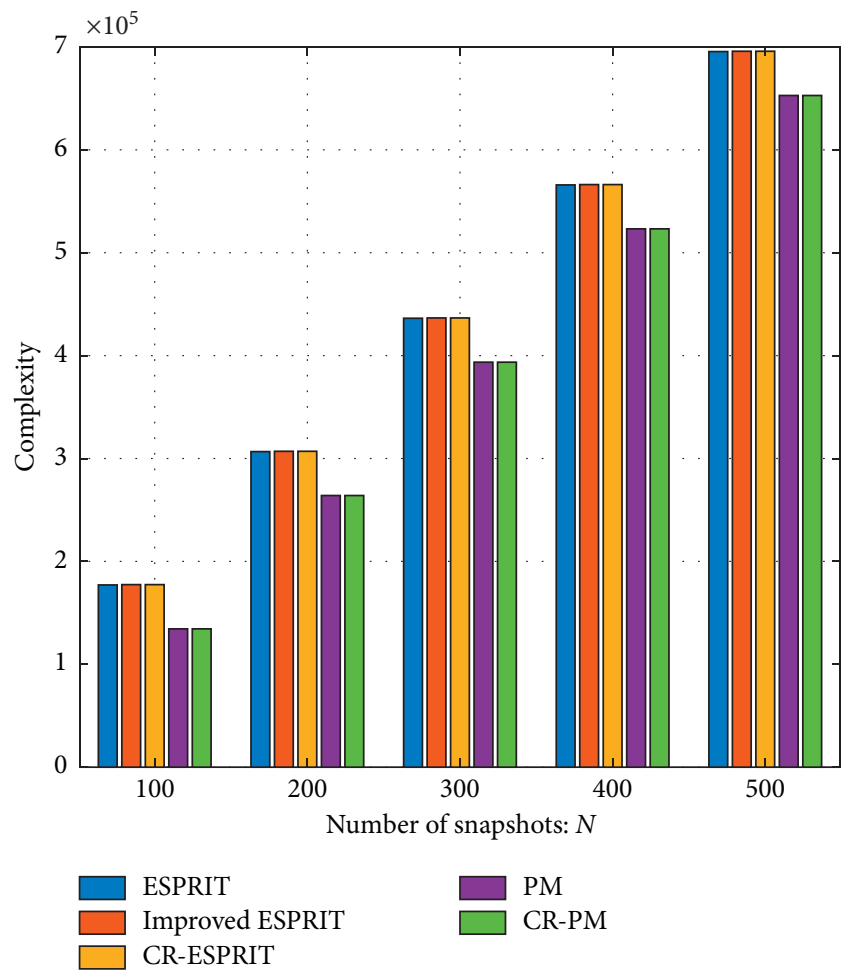

FIGURE 2: Comparison of algorithm complexity under different snapshots $N$.

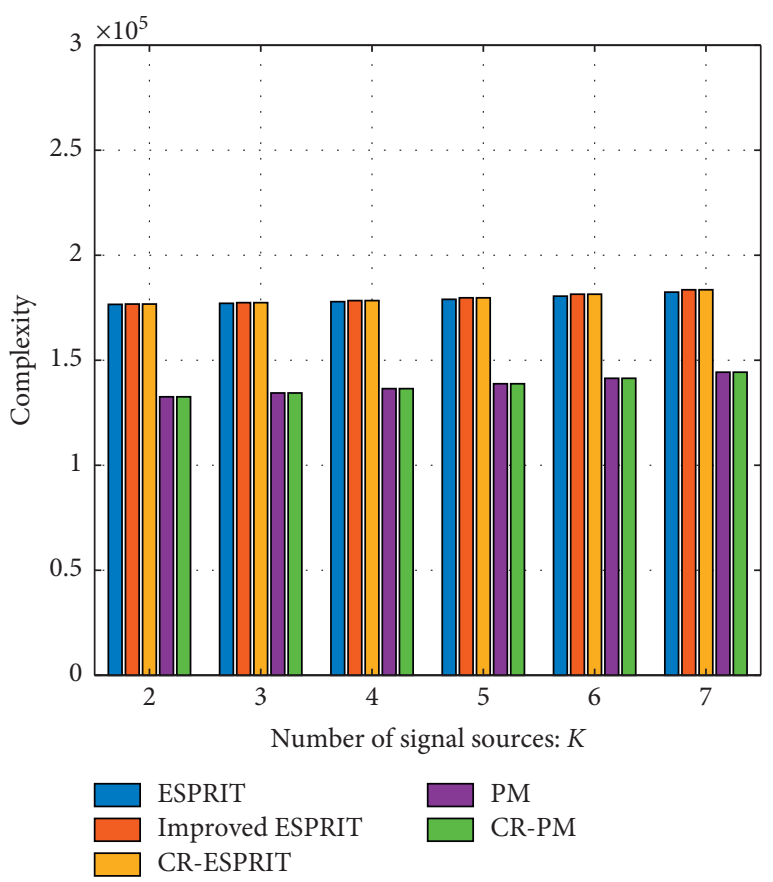

FIgURE 3: Comparison of algorithm complexity under different signal sources $K$.

3.2.3. Performance Analysis under Different Numbers of Snapshots $N$. We set $d=50 \mathrm{~m}, K=3, P=3$, and $M=12$. We also set different numbers of snapshots $(N=100,400$, and 800 ). The SNR range is from $-15 \mathrm{~dB}$ to $15 \mathrm{~dB}$ (step: $2 \mathrm{~dB}$ ),
TABLE 1: Running time of these methods.

\begin{tabular}{lc}
\hline Methods & Running time (s) \\
\hline PM & 2.3291 \\
CR-PM & 2.6634 \\
ESPRIT & 2.8223 \\
Improved ESPRIT & 2.8454 \\
CR-ESPRIT & 3.1177 \\
\hline
\end{tabular}

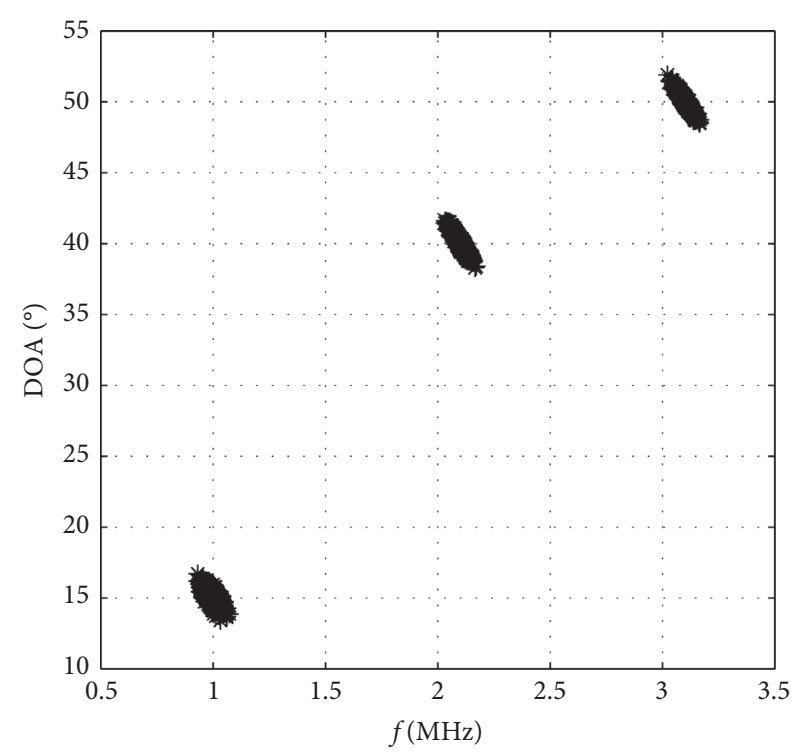

Figure 4: Parameter estimation for a uniform array.

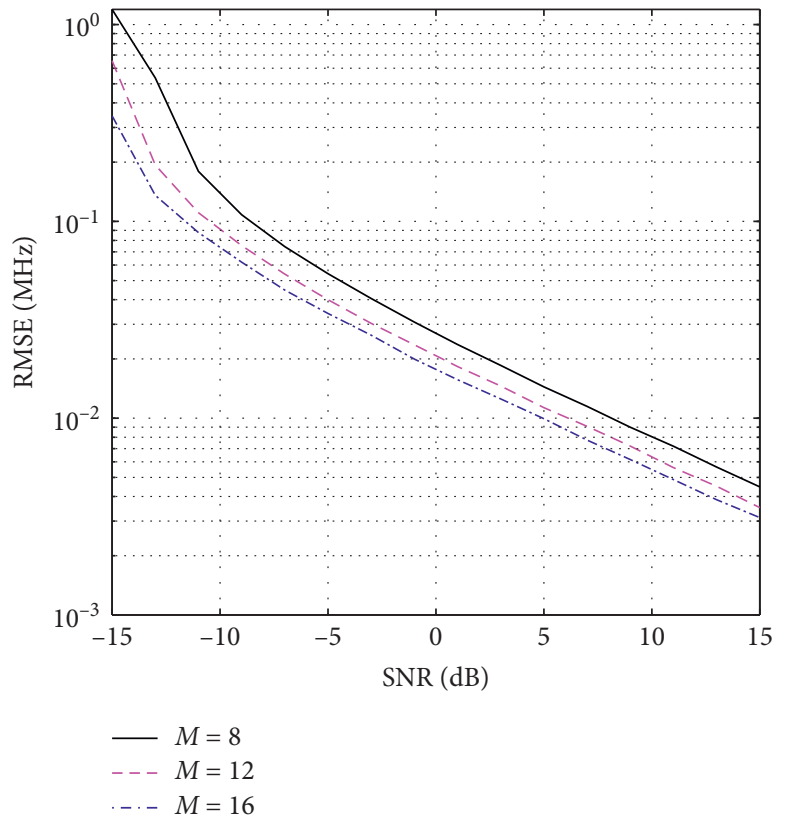

FIGURE 5: RMSEs of $f_{k}$ under different numbers of array elements.

and the RMSEs of the frequency and DOA estimations of the method proposed in this paper are shown in Figure 7 and 8, respectively. We can see from Figure 7 and 8 that when the SNR is within the range of $-15 \mathrm{~dB}$ to $15 \mathrm{~dB}$ (with steps of 


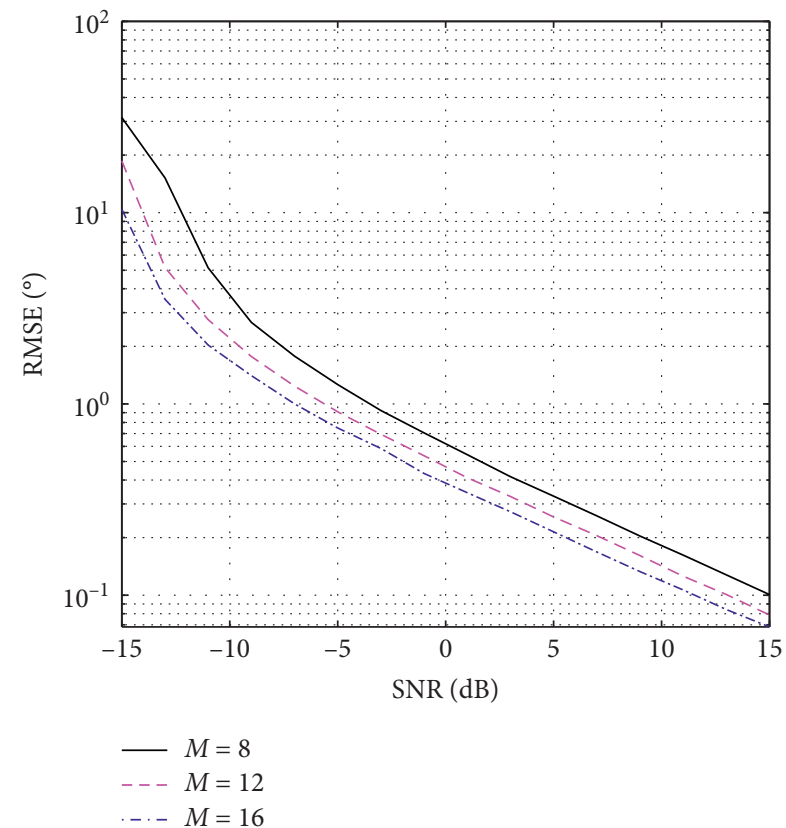

Figure 6: RMSEs of the DOA under different array elements.

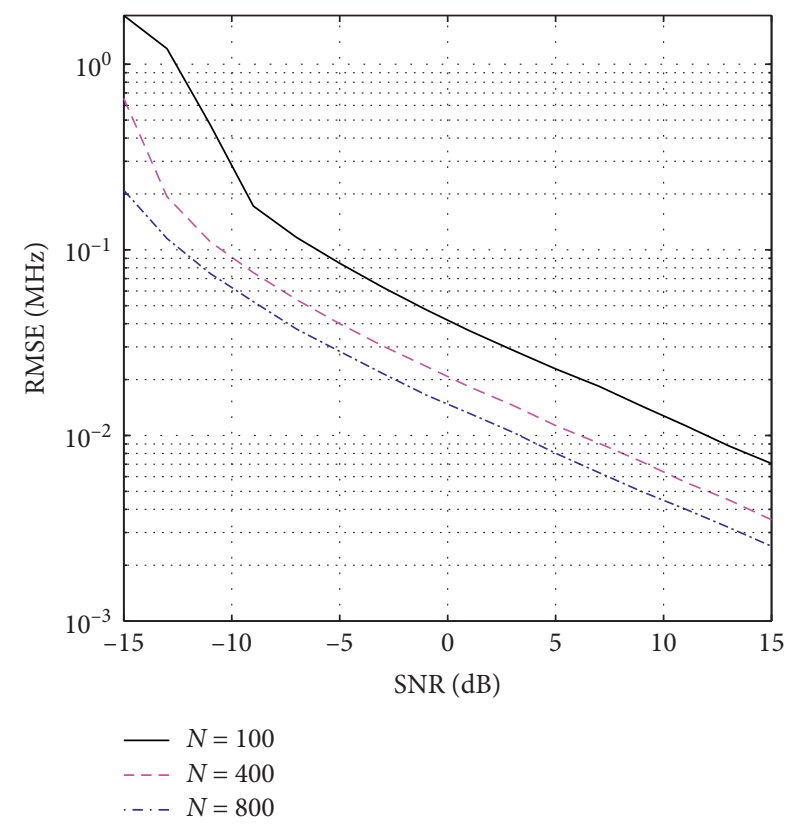

FIGURE 7: RMSEs of $f_{k}$ under different numbers of snapshots.

$2 \mathrm{~dB}$ ), the RMSEs of the proposed method demonstrate that with different snapshots, the algorithm can still maintain high estimation performance. Even in a situation with a low SNR, the estimation performance is still stable. As the number of snapshots increases, the estimation accuracy of the method proposed in this paper is enhanced, the performance is more precise, and the RMSEs of the frequency and DOA estimations of the proposed method decrease.

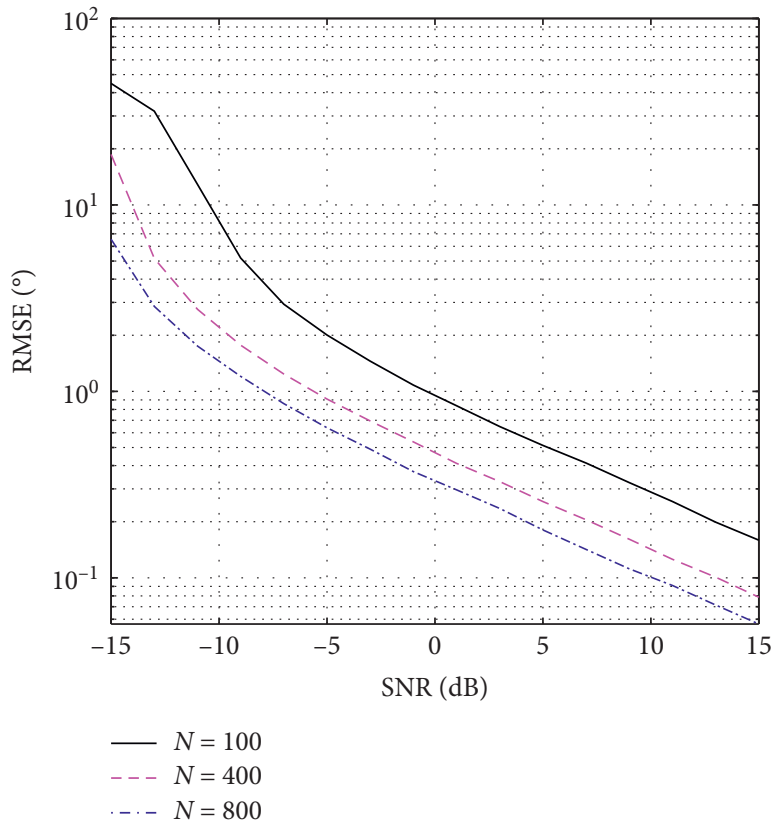

Figure 8: RMSEs of the DOA under different numbers of snapshots.

3.2.4. Performance Analysis under Different Delay Values $P$. We set $d=50 \mathrm{~m}, K=3, N=400$, and $M=12$. We also set different delay values $(P=2,3$, and 4$)$. The range of the SNR is from $-15 \mathrm{~dB}$ to $15 \mathrm{~dB}$ (step: $2 \mathrm{~dB}$ ), and the RMSEs of the frequency and DOA estimations of the method proposed in this paper are shown in Figures 9 and 10, respectively. In Figures 9 and 10, under different delay values, the proposed method maintains high DOA and frequency estimation performance when the SNR ranges from $-15 \mathrm{~dB}$ to $15 \mathrm{~dB}$. The estimation performance is stable even in a situation with a low SNR. As the delay value increases, the estimation accuracy of the method proposed in this paper is enhanced, the performance is more precise, and the RMSEs of the DOA and frequency estimations of the method decrease.

3.2.5. Performance Analysis under Different Numbers of Signal Sources K. We set $d=50 \mathrm{~m}, P=3, N=400$, and $M=12$. We also set different numbers of signal sources $(K=2,3$, and 4$)$. The range of the SNR is from $-15 \mathrm{~dB}$ to $15 \mathrm{~dB}$ (step: $2 \mathrm{~dB}$ ), and the RMSEs of the frequency and DOA estimations of the method proposed in this paper are shown in Figures 11 and 12, respectively. In Figures 11 and 12, under different numbers of signal sources, the proposed method maintains high DOA and frequency estimation performance when the SNR ranges from $-15 \mathrm{~dB}$ to $15 \mathrm{~dB}$. The estimation performance is stable even in a situation with a low SNR. As the number of signal sources increases, the estimation accuracy of the method proposed in this paper makes the performance more imprecise, and the RMSEs of the DOA and frequency estimations of the proposed method increase. As the number of signal sources increases, the 


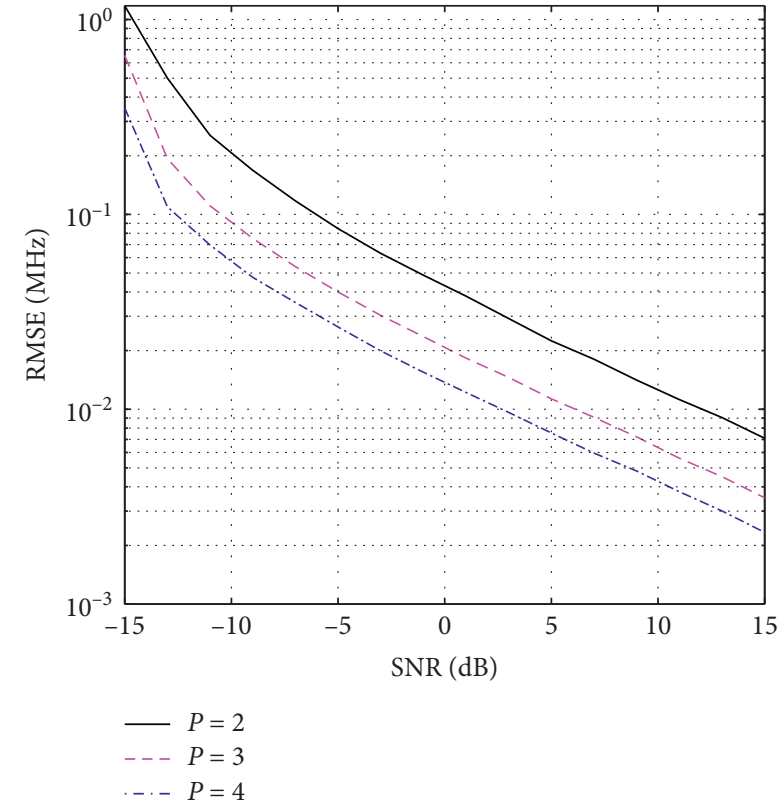

FIGURE 9: RMSEs of $f_{k}$ under different delay values.

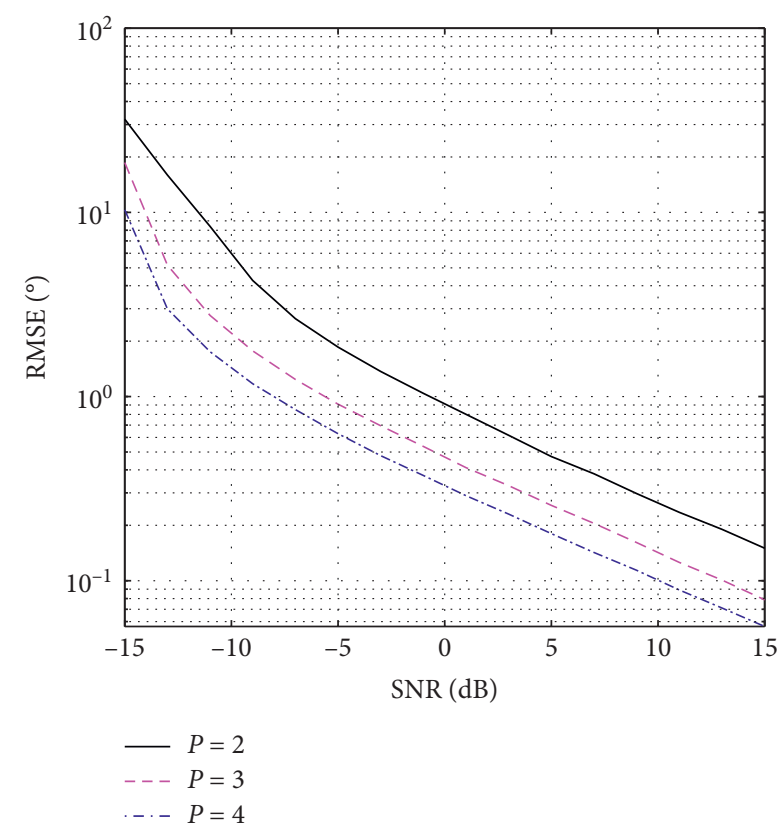

FIGURE 10: RMSEs of the DOA under different delay values.

interference between sources increases, and the frequency and DOA estimation performances deteriorate [30].

3.2.6. Identification Performance Analysis for Signal Sources with Close Angles. We set $d=50 \mathrm{~m}, K=2, P=4, N=200$, and $M=12$. In this section, we focus on exploring the recognition and identification abilities of the proposed method when the signal sources are at relatively close angles. The DOAs and operating frequencies of the signals are $\left(\theta_{1}, f_{1}\right)$ $=\left(15^{\circ}, 1 \mathrm{MHz}\right)$ and $\left(\theta_{2}, f_{2}\right)=\left(17^{\circ}, 2.1 \mathrm{MHz}\right)$. We also set

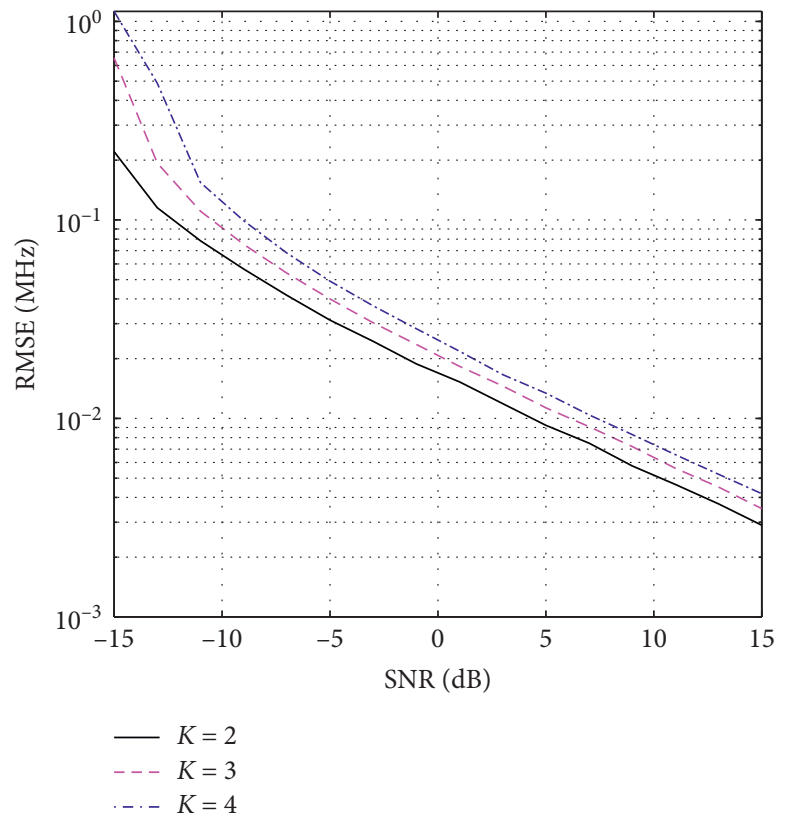

FIGURE 11: RMSEs of $f_{k}$ under different numbers of signal sources.

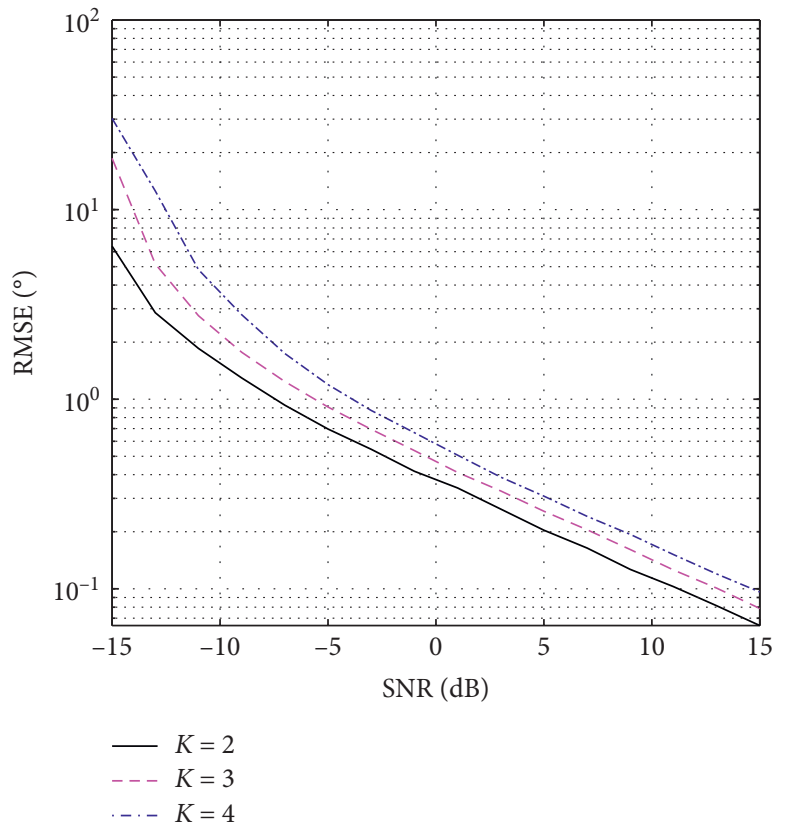

FIgURE 12: RMSEs of the DOA under different numbers of signal sources.

$\mathrm{SNR}=5 \mathrm{~dB}$. As shown in Figure 13, for signal sources with close angles, the proposed method can also perform effective identification and parameter estimation.

3.2.7. Performance Analysis of the Proposed Method in a Nonuniform Array. In an actual field receiving system, the assumed reception model is different from the true model even after a calibration procedure [31]. Therefore, in this section, we mainly discuss the performance analysis under 


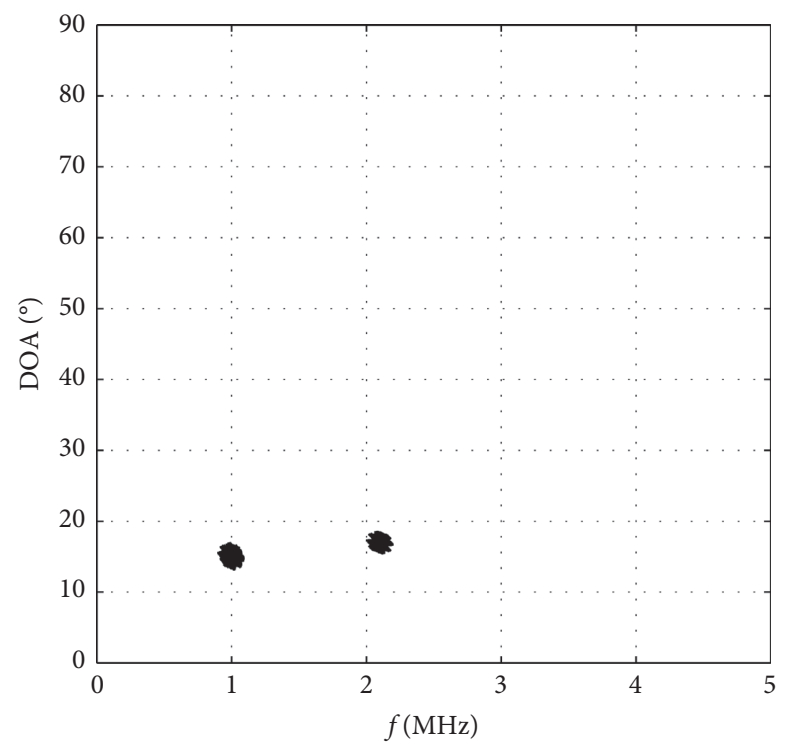

Figure 13: The identification ability of the proposed method.

the condition of a nonuniform array, such as array element position deviation [32] and uneven distance between array elements.

In this section, we assume that the array receives signals emitted by three incoherent far-field sources. The DOAs and operating frequencies of the signals are $\left(\theta_{1}, f_{1}\right)=\left(15^{\circ}\right.$, $1 \mathrm{MHz}), \quad\left(\theta_{2}, f_{2}\right)=\left(20^{\circ}, 1.9 \mathrm{MHz}\right)$, and $\left(\theta_{3}, f_{3}\right)=\left(30^{\circ}\right.$, $2.8 \mathrm{MHz}$ ). $\mathrm{SNR}=0 \mathrm{~dB}, M=12, P=5, N=300$, and $K=3$. For analyzing array element position deviation, we set $d=[0 ; 50$; $101 ; 149 ; 200 ; 251 ; 300 ; 352 ; 401 ; 449 ; 501 ; 548] m$. For uneven distance between array elements, we set $d=[0 ; 40$; $100 ; 150 ; 195 ; 235 ; 310 ; 365 ; 395 ; 450 ; 500 ; 540] \mathrm{m}$. The scatter diagram of the joint frequency and DOA estimation of the proposed method in this paper is shown in Figures 14 and 15. Figures 14 and 15 show that the proposed method is efficient in estimating the frequency and DOA results for both nonuniform array conditions.
3.2.8. Analysis of the Performances of Different Methods. In this section, we focus on analyzing the performances of different methods. We assume that the array receives signals emitted by two incoherent far-field sources. The DOAs and operating frequencies of the signals are $\left(\theta_{1}, f_{1}\right)=\left(15^{\circ}, 1 \mathrm{MHz}\right)$ and $\left(\theta_{2}, f_{2}\right)=\left(40^{\circ}, 2.1 \mathrm{MHz}\right)$. We set $d=50 \mathrm{~m}, K=2, P=2$, $N=400$, and $M=12$. The range of the SNR is from $-15 \mathrm{~dB}$ to $15 \mathrm{~dB}$ (step: $2 \mathrm{~dB}$ ), and the RMSEs of the Cramer-Rao lower bound (CRLB), the PM, the CR-PM, the ESPRIT method [16], the improved ESPRIT method [20], and the method proposed in this paper with respect to the frequency and DOA estimations are shown in Figures 16 and 17, respectively.

To quantitatively illustrate, under the condition of a low SNR, compared with the improved ESPRIT method [20], the estimate improvement of the method proposed in this paper, we define the relative improvement ratio as

$$
\begin{aligned}
\text { ratio }_{\text {frequency }} & =1-\frac{\mathrm{RMSE}_{\text {frequency }}(\mathrm{CR}-\mathrm{ESPRIT})}{\mathrm{RMSE}_{\text {frequency }}(\text { Improved ESPRIT })} \times 100 \%, \\
\text { ratio }_{\mathrm{DOA}} & =1-\frac{\mathrm{RMSE}_{\mathrm{DOA}}(\mathrm{CR}-\mathrm{ESPRIT})}{\mathrm{RMSE}_{\mathrm{DOA}}(\text { Improved ESPRIT })} \times 100 \% .
\end{aligned}
$$

According to the definitions of equations (35) and (36), we show the relative improvement ratio in Figures 18 and 19.

As shown in Figures 16 and 17, when the SNR is within the range of $-15 \mathrm{~dB}$ to $15 \mathrm{~dB}$ (in steps of $2 \mathrm{~dB}$ ), the estimation accuracy of the proposed method is better than that of the PM, the CR-PM, the ESPRIT method [16], and the improved ESPRIT method [20] in terms of both the DOA and frequency. Among them, the ESPRIT method has extremely poor angle estimation accuracy since it cannot automatically pair parameters.

As shown in Figures 18 and 19, when $\mathrm{SNR}=-15 \mathrm{~dB}$ to $-1 \mathrm{~dB}$, compared with the improved ESPRIT method, the estimation accuracy of the proposed method is greatly improved. In particular, when $\mathrm{SNR}=-15 \mathrm{~dB}$, compared with the improved ESPRIT method, the frequency estimation accuracy of the method proposed in this paper is an approximately $25.50 \%$ improvement; the DOA estimation 


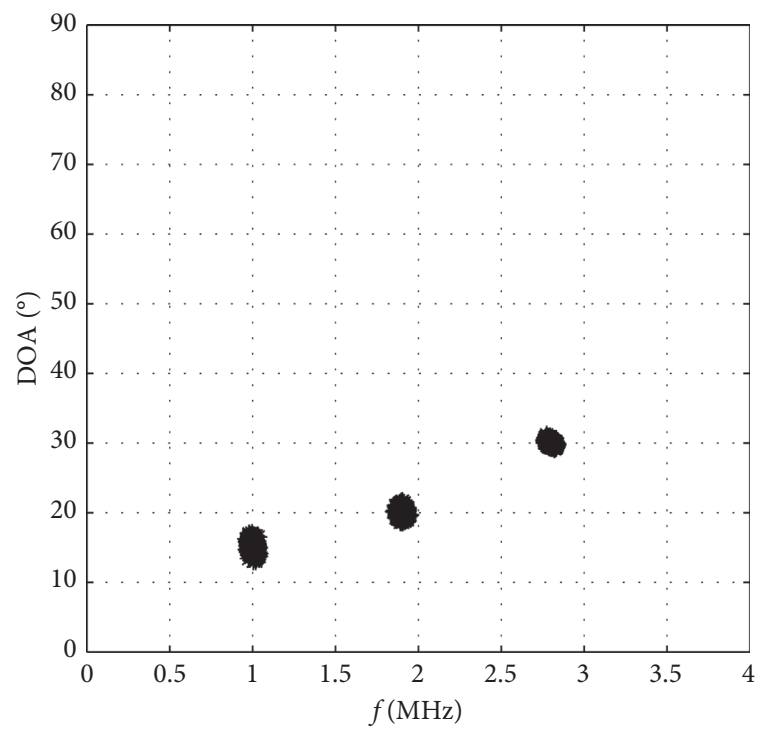

Figure 14: Parameter estimation for array element position deviation.

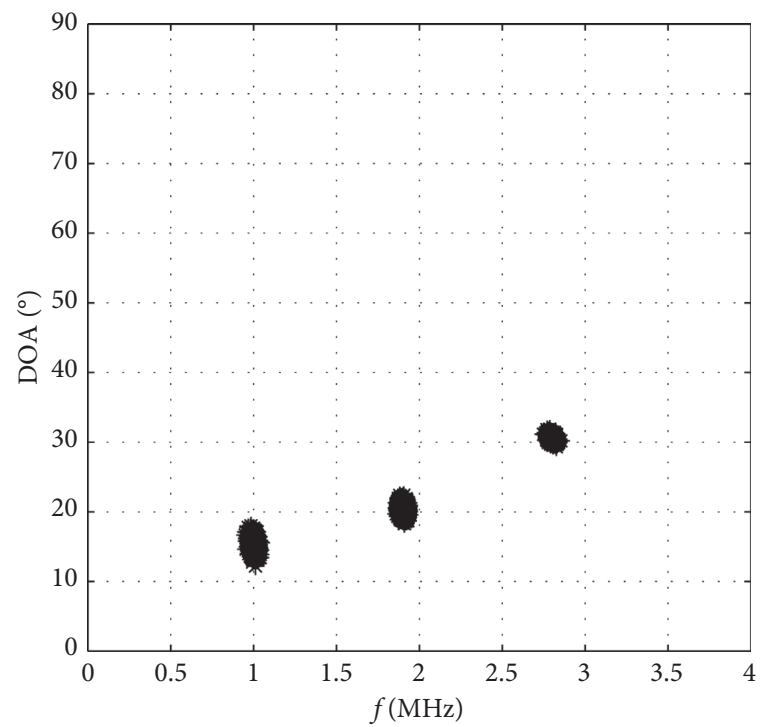

FIGURE 15: Parameter estimation for uneven distances between array elements.

accuracy of the method proposed in this paper is an approximately $31.95 \%$ improvement. However, when $\mathrm{SNR}=-1 \mathrm{~dB}$ to $15 \mathrm{~dB}$, the relative improvement ratio fluctuates around zero. This result illustrates that the estimation accuracy of the proposed method is almost the same as that of the improved ESPRIT method. For fluctuation, we surmise that the reason for this phenomenon may be the result of too few simulations in this paper.

In summary, a comprehensive analysis of Figures 16-19 shows that the estimation accuracy of the proposed method is improved over that of the PM, the CR-PM, the ESPRIT

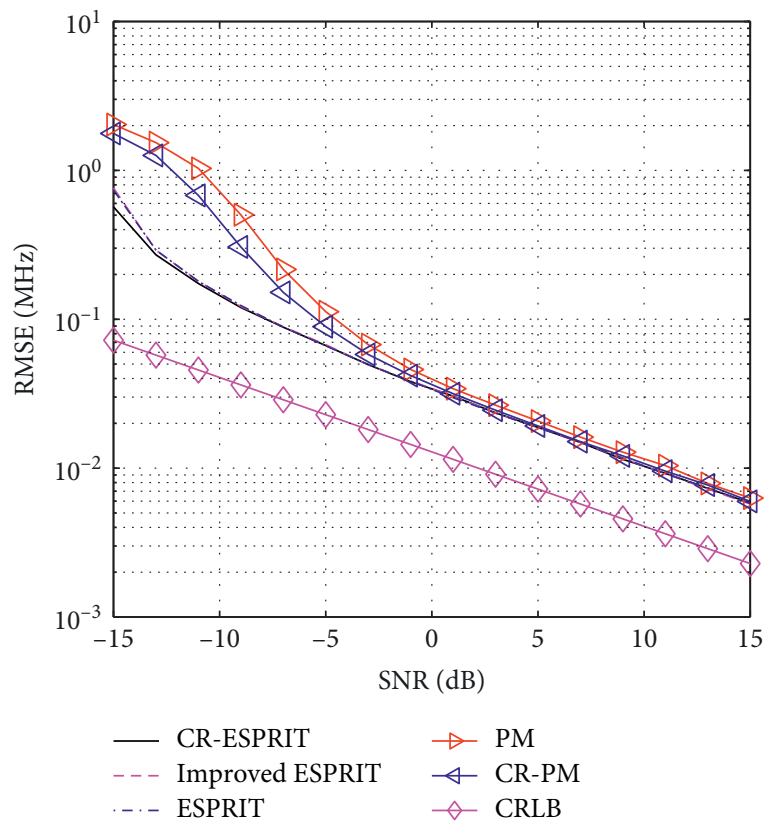

FIGURE 16: RMSEs of $f_{k}$ under different methods.

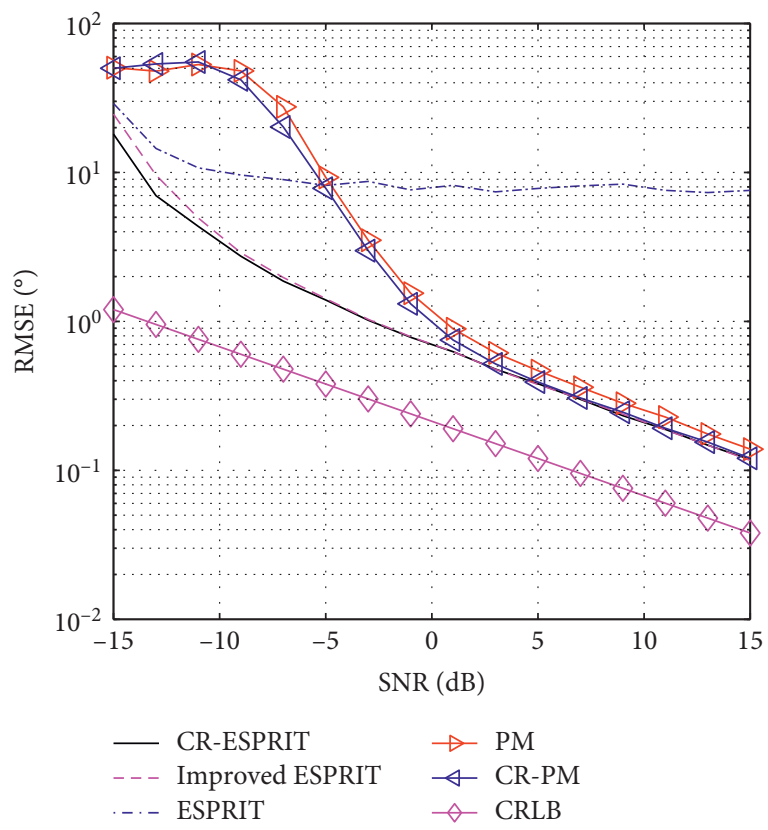

Figure 17: RMSEs of the DOA under different methods.

method, and the improved ESPRIT method. The results further verify that the method proposed in this paper has good anti-noise performance and stability under different SNRs. Therefore, compared to the PM, the CR-PM, the ESPRIT method, and the improved ESPRIT method, the method proposed in this paper is more suitable for use in a complex electromagnetic environment. 


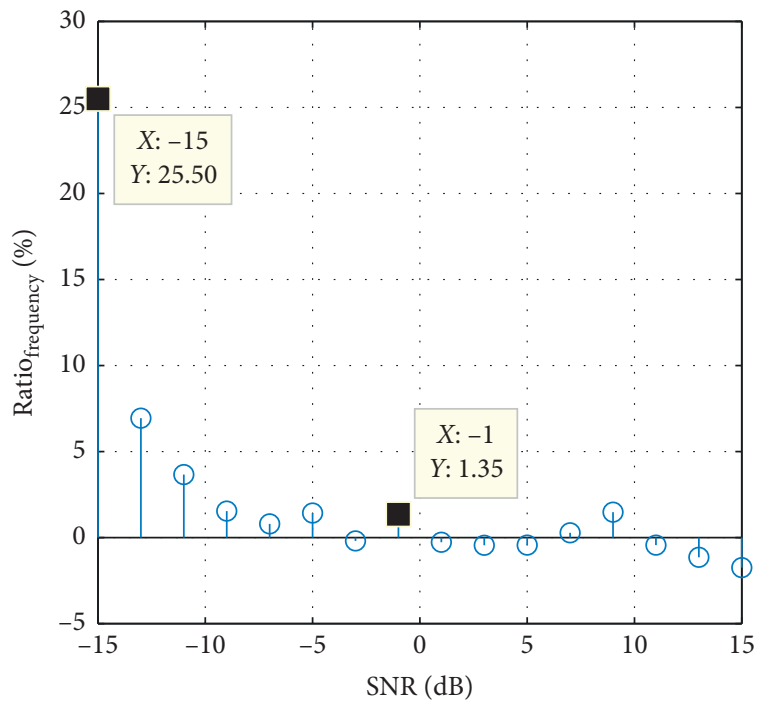

FIGURE 18: The relative improvement ratios of the frequency estimation.

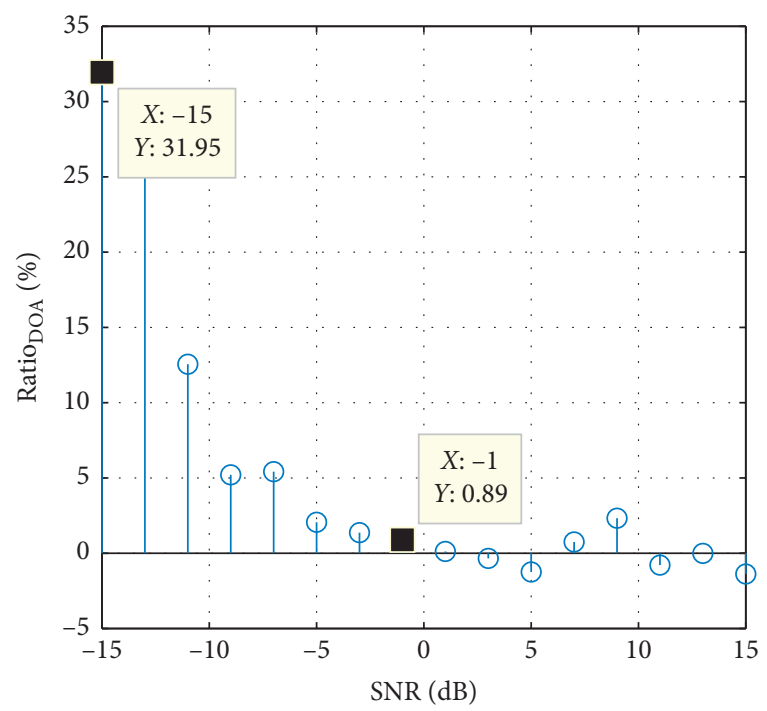

FIGURE 19: The relative improvement ratios of the DOA estimation.

\section{Conclusions}

For linear arrays, this paper proposes a joint angle and frequency estimation method based on CR-ESPRIT. We first preprocess the received signal by taking full advantage of the conjugate information contained in the originally received data, and we reconstruct a new total covariance matrix. Then, we use the LS-ESPRIT algorithm to estimate the frequency parameter. According to the unique relationship between angles and frequencies, we estimate the DOAs based on the reconstructed received signal. The complexity of the method proposed in this paper is almost the same as that of the ESPRIT and the improved ESPRIT. Numerical simulations and comparisons with the PM, the CR-PM, the ESPRIT method, and the improved ESPRIT method prove the superiority of the proposed method. In a real space environment, under the condition of a uniform or a nonuniform array, this method can realize the automatic pairing of the estimated DOAs and frequencies of radiation source signals without an additional parameter pairing process. Moreover, this method has high accuracy and strong anti-noise performance when conducting parameter estimation.

\section{Data Availability}

The data used to support the findings of the study are included within this paper.

\section{Conflicts of Interest}

The authors declare that there are no conflicts of interest regarding the publication of this paper.

\section{Acknowledgments}

The authors would like to thank Prof. M. H. PAN for his support and funding for this project. The authors thank C. Wang and C. B. Ye for their help. This work was supported by the National Natural Science Foundation of China under Grant no. 61671241.

\section{References}

[1] J.-D. Lin, W.-H. Fang, Y.-Y. Wang, and J.-T. Chen, "FSF MUSIC for joint DOA and frequency estimation and its performance analysis," IEEE Transactions on Signal Processing, vol. 54, no. 12, pp. 4529-4542, 2006.

[2] J. R. Jensen, M. G. Christensen, and S. H. Jensen, "Nonlinear least squares methods for joint DOA and pitch estimation," IEEE Transactions on Audio, Speech, and Language Processing, vol. 21, no. 5, pp. 923-933, 2013.

[3] V. Varadarajan and J. Krolik, "Array shape estimation and tracking using active sonar reverberation," IEEE Transactions on Aerospace and Electronic Systems, vol. 40, no. 3, pp. 1073-1086, 2004.

[4] J. Wang, G. Ding, Q. Wu, L. Shen, and F. Song, "Spatialtemporal spectrum hole discovery: a hybrid spectrum sensing and geolocation database framework," Chinese Science Bulletin, vol. 59, no. 16, pp. 1896-1902, 2014.

[5] X. Zeng, B. Tang, and Y. Xiong, "Interception algorithm of S-cubed signal model in stealth radar equipment," Chinese Journal of Aeronautics, vol. 25, no. 3, pp. 416-422, 2012.

[6] G. Hu, Y. Zhang, M. Jing, Y. Tang, and B. Gu, "Credibility test for blind processing results of sinusoid using Chebyshev's Inequality," in Proceedings of the 2013 IEEE International Conference on Signal Processing, Communication and Computing (ICSPCC 2013), pp. 1-6, IEEE, Kunming, China, August 2013.

[7] G. Hu, P. Zhao, Z. Chen, and R. Luo, "Confidence evaluation for blind analysis of LFM/BPSK hybrid signals based on extreme value theory," IET Radar, Sonar \& Navigation, vol. 13, no. 3, pp. 473-482, 2018.

[8] G. Hu and P. Zhao, "Confidence test for blind analysis of BPSK signals," IEEE Transactions on Aerospace and Electronic Systems, vol. 55, no. 2, pp. 658-675, 2019.

[9] M. A. Hannan, P. Rocca, and A. Massa, "Robust BCS-based direction-of-arrival and bandwidth estimation of unknown signals for cognitive radar," in Proceedings of the 2018 IEEE International Symposium on Antennas and Propagation and 
USNC/URSI National Radio Science Meeting, pp. 625-626, IEEE, Boston, MA, USA, May 2018.

[10] M. Zuo, S. Xie, Y. Li, and C. Zhang, "Joint estimation method for frequency and DOA of virtual antenna array in space-time domain," in Proceedings of the 2019 International Applied Computational Electromagnetics Society Symposium - China (ACES), pp. 1-2, IEEE, Nanjing, China, August 2019.

[11] X. Huang, L. Cao, and A. Liu, "Sequential estimation of frequency and direction-of-arrival based on the relaxed coprime array for multiple targets," IEEE Access, vol. 8, pp. 80318-80332, 2020.

[12] X. Zhang, L. Xu, L. Xu, and D. Xu, "Direction of departure (DOD) and direction of arrival (DOA) estimation in MIMO radar with reduced-dimension MUSIC," IEEE Communications Letters, vol. 14, no. 12, pp. 1161-1163, 2010.

[13] X. Zhang and D. Xu, "Angle estimation in bistatic MIMO radar using improved reduced dimension Capon algorithm," Journal of Systems Engineering and Electronics, vol. 24, no. 1, pp. 84-89, 2013.

[14] C. Wang, Research on Identification and Parameter Estimation of Intra-pulse Modulated Signals at Low SNR, Northeast Normal University, Changchun, China, 2019.

[15] S. Sirianunpiboon, S. D. Howard, and S. D. Elton, "Timedecentralized DOA estimation for electronic surveillance," in Proceedings of the 2017 51st Asilomar Conference on Signals, Systems, and Computers, pp. 1137-1141, IEEE, Pacific Grove, CA, USA, June 2017.

[16] X. Wang, "Joint angle and frequency estimation using multiple-delay output based on ESPRIT," EURASIP Journal on Advances in Signal Processing, vol. 2010, no. 1, Article ID 358659, 2010.

[17] R. Schmidt, "Multiple emitter location and signal parameter estimation," IEEE Transactions on Antennas and Propagation, vol. 34, no. 3, pp. 276-280, 1986.

[18] A. N. Lemma, A. van der Veen, and E. F. Deprettere, "Analysis of joint angle-frequency estimation using ESPRIT," IEEE Transactions on Signal Processing, vol. 51, no. 5, pp. 12641283, 2003.

[19] Z. Sun, X. Zhang, H. Wu, and J. Liu, "Propagator methodbased joint angle and frequency estimation using multiple delay output," ICIC Express Letters, vol. 2, no. 4, pp. 827-832, 2011.

[20] X. Wang, X. Zhang, J. Li, and J. Bai, "Improved ESPRIT method for joint direction-of-arrival and frequency estimation using multiple-delay output," International Journal of Antennas and Propagation, vol. 2012, pp. 1018-1020, 2012.

[21] Y. Gao, Y. Ma, S. Li, X. Zhou, and S. Wu, "A novel joint angle and frequency estimation method based on the extended OMP algorithm," in Proceedings of the 2019 IEEE Globecom Workshops (GC Wkshps), pp. 1-5, IEEE, Waikoloa, HI, USA, May 2019.

[22] L. Xu, F. Wen, and X. Zhang, "A novel unitary PARAFAC algorithm for joint DOA and frequency estimation," IEEE Communications Letters, vol. 23, no. 4, pp. 660-663, 2019.

[23] B. E. Nazarenko, T. K. Artemova, and A. S. Gvozdarev, "Linear antenna array circular bending effect on the impedance matrix and input impedances' unbalance," in Proceedings of the 2020 International Youth Conference on Radio Electronics, Electrical and Power Engineering (REEPE), pp. 1-5, IEEE, Moscow, Russia, July 2020.

[24] A. A. Kumar, S. G. Razul, M. G. Chandra, C. M. See, and P. Balamuralidhar, "Joint frequency and direction of arrival estimation with space-time array," in Proceedings of the 2016 IEEE Sensor Array and Multichannel Signal Processing
Workshop (SAM), pp. 1-5, IEEE, Rio de Janeiro, Brazil, October 2016.

[25] L. Xu, R. Wu, X. Zhang, and Z. Shi, "Joint two-dimensional DOA and frequency estimation for L-shaped array via compressed sensing PARAFAC method," IEEE Access, vol. 6, pp. 37204-37213, 2018.

[26] L. Xu, R. Wu, X. Zhang, and Z. Shi, "Efficient 2-D DOA and frequency estimation for L-shaped array via RD-PM," International Journal of Electronics, vol. 106, no. 9, pp. 13941411, 2019.

[27] S. Zheng, X. Zhang, B. Zong, and J. Xu, "Parameter estimation of the 3D-GTD model based on a modified 3D-ESPRIT algorithm," Journal of Beijing University of Posts and Telecommunications, vol. 43, no. 4, pp. 32-38, 2020.

[28] S. Zheng, X. Zhang, Y. Guo, B. Zong, and J. Xu, "Parameter estimation of 1D GTD scattering center model based on an improved MUSIC algorithm," Journal of Beijing University of Aeronautics and Astronautics, vol. 46, no. 11, pp. 2149-2155, 2020.

[29] L. Xu, R. Wu, and X. Zhang, "2D-DOA and frequency estimation for L-shaped array via reduced-dimensional MUSIC," Systems Engineering and Electronics, vol. 41, no. 1, pp. 1-8, 2019.

[30] C. Chen and X. Zhang, "A coherent direction of arrival estimation method using a single pulse," Computers and Electrical Engineering, vol. 40, no. 5, pp. 1731-1740, 2014.

[31] A. Ferréol, P. Larzabal, and M. Viberg, "Statistical analysis of the MUSIC algorithm in the presence of modeling errors, taking into account the resolution probability," IEEE Transactions on Signal Processing, vol. 58, no. 8, pp. 4156-4166, 2010.

[32] Y. Tian, J. X. Shi, and Y. R. Wang, "Direction of arrival estimation method using partly calibrated nested array in the presence of gain-phase errors," Acta Electronica Sinica, vol. 47, no. 12, pp. 2465-2471, 2019. 